\title{
Note on the effective choice of a point in the complement of an analytic set
}

\author{
By Yoemon SAMPeI
}

(Received March 25, 1961)

In their paper [1], N. Lusin and P. Novikoff gave a method of the effective choice of a point in the complement of an analytic set. In the present paper, we shall note that, modifying the process of $\mathrm{N}$. Lusin and P. Novikoff by imposing various requirements, we can obtain those processes which enable us to choose a point, in the complement of an analytic set, satisfying some supplementary conditions.

1. A generalization of Lusin-Novikoff's theorem. Let $E$ be the complement of an analytic set in the space of all irrational numbers $x$, $0<x<1$. Assume that the set $E$ is defined by means of a sieve

$$
C=\sum_{n=1}^{\infty} \delta_{n} \times\left\{r_{n}\right\}
$$

where

$$
\delta_{1}, \delta_{2}, \cdots, \delta_{n}, \cdots
$$

are Baire's intervals and $r_{n}, n=1,2, \cdots$, are rational numbers. Assume moreover that the sieve $C$ satisfies the condition:

$1^{\circ}$ If any two of Baire's intervals $\delta_{n_{1}}, \delta_{n_{2}}, \cdots, \delta_{n_{k}}, \cdots$ are not disjoint, then the set $\prod_{i=1}^{\infty} \delta_{n_{i}}$ consists of exactly one point.

This condition is obviously satisfied, if no Baire's interval appears infinitely many times in (2).

Providing for the necessity in setions 2 and 3, we assume moreover:

$2^{\circ} \quad P_{x} C$ is countable for every $x$, where $P_{x}$ is the perpendicular to the $x$-axis at $x$.

Let $C_{n}$ denote the sieve, consisting of all those points $x \in C$ which lie strictly below the set $\delta_{n} \times\left\{r_{n}\right\}$. We shall denote order types of $P_{x} C$ and $P_{x} C_{n}$ by $\nu(x)$ and $\nu_{n}(x)$ respectively.

We have

Lemma. Let $E^{(i-1)}, n_{i}$ and $\gamma_{i}, i=1,2, \cdots$, be respectively a sequence of subsets of the interval $0<x<1$, that of natural numbers and that of ordinal numbers. Assume

1) $E^{(i-1)} \neq 0$,

2) $E \supseteqq E^{(i-1)} \supseteqq E^{(i)}$, 
3) $n_{i}$ is the smallest $n$ such that $n>n_{i-1}$ and $\delta_{n} E^{(i-1)} \neq 0$,

4) if $x \in E^{(i)}$ then $\xi_{n_{i}}(x)=\gamma_{i}$,

5) $E^{(\mathfrak{)})} \subseteq \delta_{n_{i}}$,

for every $i=1,2, \cdots$.

Then, the intervals $\delta_{n_{1}}, \delta_{n_{2}}, \cdots, \delta_{n_{k}}, \cdots$ have exactly one point $\xi=\prod_{i=1}^{\infty} \delta_{n_{i}}$ in common. Among the intervals in (2), $\delta_{n_{1}}, \delta_{n_{2}}, \cdots, \delta_{n_{k}}, \cdots$ are only intervals containing the point $\xi$. Finally, $\xi$ is contained in $E$.

Proof. It follows from 2) that $E^{(i)} \supseteqq E^{(j)}$ when $i<j$. Then, 5) and 1) imply that $\delta_{n_{i}} \delta_{n_{j}} \neq 0$. Hence, on account of $1^{\circ}$, it follows that the intervals $\delta_{n_{k}}, k=1,2, \cdots$, have exactly one common point $\xi=\prod_{i=1}^{\infty} \delta_{n_{i}}$.

Suppose that $n_{i-1}<n \leqq n_{i}$ and $\xi \in \delta_{n}$. On account of the fact that $\xi=\prod_{i=1}^{\infty} \delta_{n_{i}}$, we have a $k$ such that $k \geqq i$ and $\delta_{n} \supseteqq \delta_{n_{k}}$. Then, from 5), 3) and 2), it follows that $\delta_{n} E^{(i-1)} \neq 0$. Hence $n=n_{i}$.

Suppose that $i<j$. Take a point $x \in E^{(j)}$. Then, it follows from 2) that $x \in E^{(i)}$. Hence $x \in \delta_{n_{i}}$ and $x \in \delta_{n_{j}}$. Therefore,

$$
r_{n_{i}}>r_{n_{j}} \text { if and only if } \nu_{n_{i}}(x)>\nu_{n_{j}}(x) \text {, }
$$

or, on account of 4 ),

$$
r_{n_{i}}>r_{n_{j}} \text { if and only if } \gamma_{i}>\gamma_{j} .
$$

This shows that the set $P_{\xi} C$ is well-ordered. Hence $\xi \in E$.

2. Effective choice of a point in a set of class $A_{\rho}$. Let $A$ be an analytic set given as a nucleus of a regular system $\left\{F_{m_{1} m_{2} \cdots m_{k}}\right\}$ of Souslin, of closed sets. Put

$$
A^{m_{1} m_{2} \cdots m_{k}}=\sum_{\left(n_{1}, n_{2}, \cdots, n_{l}, \cdots\right)} \prod_{l=1}^{\infty} F_{m_{1} m_{2} \cdots m_{k} n_{1} n_{2} \cdots n_{l}}
$$

Let $E$ be the complement of an analytic set, defined by means of a sieve (1) with the properties $1^{\circ}$ and $2^{\circ}$.

Assuming that $A E \neq 0$, we shall choose a point $\xi$ in $A E$.

The choice-process consists of the following steps.

First-STEP. Let $E_{\gamma}$ be the first constituent of $E$, defined by $C$, such that $A E_{\gamma} \neq 0$. Put $E^{(0)}=A E_{\gamma}$.

We define $n_{1}, H_{1}, \gamma_{1}, K_{1}, m_{1}$ and $E^{(1)}$ in the following way:

$n_{1}$ is the smallest $n$ such that $\delta_{n} E^{(0)} \neq 0$,

$$
H_{1}=\delta_{n_{1}} E^{(0)} \text {, }
$$


$\gamma_{1}$ is the minimum of all $\nu_{n_{1}}(x), x \in H_{1}$,

$K_{1}$ is the set of all $x \in H_{1}$ such that $\nu_{n_{1}}(x)=\gamma_{1}$,

$m_{1}$ is the smallest $m$ such that $A^{m} K_{1} \neq 0$,

$E^{(1)}=A^{m_{1}} K_{1}$.

Obviously, $E^{(1)} \subseteq A^{m_{1}}$.

$k$-TH STEP. Suppose that we have already constructed those $n_{i}, H_{i}$, $\gamma_{i}, K_{i}, m_{i}$ and $E^{(i)}(i=1,2, \cdots, k-1)$ which, besides 1$\left.)-5\right)$, satisfy the condition:

6) $\quad E^{(i)} \cong A^{m_{1} m_{2} \ldots m_{i}}$,

for every $i=1,2, \cdots, k-1$.

We define $n_{k}, H_{k}, \gamma_{k}, K_{k}, m_{k}$ and $E^{(k)}$ in the following way:

$n_{k}$ is the smallest $n$ such that $\delta_{n} E^{(k-1)} \neq 0$ and $n>n_{k-1}$,

$H_{k}=\delta_{n_{k}} E^{(k-1)}$,

$\gamma_{k}$ is the minimum of all $\nu_{n_{k}}(x), x \in H_{k}$,

$K_{k}$ is the set of all $x \in H_{k}$ such that $\nu_{n_{k}}(x)=\gamma_{k}$,

$m_{k}$ is the smallest $m$ such that $A^{m_{1} m_{2} \cdots m_{k-1} m} K_{k} \neq 0$,

$E^{(k)}=A^{m_{1} m_{2} \cdots m_{k-1} m_{k}} K_{k}$.

Then, the conditions 1 ) -6 ) are obviously satisfied even when $i=k$.

Because of $2^{\circ}$, we can progress these steps indefinitely. Thus, we have infinite sequences $n_{i}, H_{i}, \gamma_{i}, K_{i}, m_{i}$ and $E^{(i-1)}$, which satisfy conditions 1)-6) for every $i=1,2, \ldots$. Hence, by Lemma, we have

$$
\xi=\prod_{i=1}^{\infty} \delta_{n_{i}} \in E .
$$

On the other hand, 1), 5) and 6) imply that

$$
\delta_{n_{i}} A^{m_{1} m_{2} \cdots m_{i}} \neq 0 .
$$

Taking into account that diameters of sets $\delta_{n_{i}}$ and $A^{m_{1} m_{2} \cdots m_{\ell}}$ tend to zero as $i \rightarrow \infty$, we have

$$
\xi=\prod_{i=1}^{\infty} A^{m_{1} m_{2} \cdots m_{i}} \in A .
$$

Moreover, taking a point $x \in E^{(i)} \cong E^{(0)} \cong E_{\gamma}$, we have $\gamma_{i}=\nu_{n_{i}}(x)<\nu(x)$ $=\gamma$. Hence $\xi \in A E_{\gamma}=E^{(0)}$.

3. A choice of a point in a constituent of the complement of an analytic set. Let $E$ be the complement of an analytic set, given by means of a sieve (1) with the properties $1^{\circ}$ and $2^{\circ}$. Let 


$$
E=E_{\omega}+E_{\omega+1}+\cdots+E_{\alpha}+\cdots
$$

be the development of $E$ into a transfinite sequence of the constituents $E_{\alpha}$, defined by $C$.

Under the assumption that an effective enumeration

$$
\alpha_{1}, \alpha_{2}, \cdots, \alpha_{n}, \cdots
$$

of the non-zero ordinal numbers less than $\alpha_{0}$ is given, we shall choose a point $\xi$ in $E_{\alpha_{0}}$. Of course, it is assumed that $E_{\alpha_{0}} \neq 0$.

The process of choice is as follows:

First-SteP. Put $E^{(0)}=E_{\alpha_{0}}$. We define $n_{1}, H_{1}, \gamma_{1}$ and $K_{1}$ in the same way as in 2. Moreover, we define

$m_{1}$ as the smallest $m$ such that $\nu_{m}(x)=\alpha_{1}$ for some $x \in K_{1}$, $E^{(1)}$ as the set of all $x \in K_{1}$ such that $\nu_{m_{1}}(x)=\alpha_{1}$.

Besides 1)-5), the conditions

7) $E^{(i)} \cong \delta_{m_{i}}$,

8) if $x \in E^{(i)}$, then $\nu_{m_{i}}(x)=\alpha_{i}$,

are satisfied for $i=1$.

$k$-TH STEP. Suppose that we have already constructed those $n_{i}, H_{i}$, $\gamma_{i}, K_{i}, m_{i}$ and $E^{(i)}(i=1,2, \cdots, k-1)$ which satisfy the conditions 1$\left.)-5\right)$ and 7); 8) for $i=1,2, \cdots, k-1$.

The definition of $n_{k}, H_{k}, \gamma_{k}$ and $K_{k}$ are the same as in 2. We define

$m_{k}$ as the smallest $m$ such that $\nu_{m}(x)=\alpha_{k}$ for some $x \in K_{k}$, $E^{(k)}$ as the set of all $x \in K_{k}$ such that $\nu_{m_{k}}(x)=\alpha_{k}$.

The conditions 1)-5) and 7), 8) are satisfied even when $i=k$.

Progressing these steps indefinitely, we have infinite sequences $n_{i}$, $H_{i}, \gamma_{i}, K_{i}, m_{i}$ and $E^{(i-1)}(i=1,2, \cdots)$, satisfying the conditions 1$\left.)-5\right)$ and $7), 8)$ for every $i=1,2, \cdots$.

It follows from Lemma that:

$$
\xi=\prod_{i=1}^{\infty} \delta_{n_{i}} \in E .
$$

Taking $x \in E^{(i)} \cong E^{(0)}$, we have

$$
\gamma_{i}=\nu_{n_{i}}(x)<\nu(x)=\alpha_{0} .
$$

On the other hand, we have, by 5) and 7), that

$$
\xi=\prod_{i=1}^{\infty} \delta_{n_{i}} \in \delta_{m_{\jmath}}, \quad j=1,2, \cdots .
$$


Hence, by Lemma, each of $\delta_{m_{1}}, \delta_{m_{2}}, \cdots, \delta_{m_{j}}, \cdots$ coincides with some $\delta_{n_{i}}$. Suppose that $\delta_{m_{j}}=\delta_{n_{i}}$. Then, taking a point $x$ in some $E^{(k)}, k \geqq n_{i}$, $k \geqq m_{j}$, we have

$$
\alpha_{j}=\nu_{m_{j}}(x)=\nu_{n_{i}}(x)=\gamma_{i} .
$$

Therefore, every $\alpha_{j}$ coincides with some $\gamma_{i}$.

Thus, we conclude that the sequence $\gamma_{1}, \gamma_{2}, \cdots, \gamma_{i}, \cdots$ consists of all ordinal numbers less than $\alpha_{0}$. Hence $\xi \in E^{(0)}$.

Summing up, we have:

Given an effective enumeration of the ordinal numbers less that $\alpha_{0}$, we can choose effectively a point in the $\alpha_{0}$-th constituent (if it is nonvoid) of the complement of an analytic set.

The converse is also true. For an effectively chosen point $\xi \in E_{\alpha_{0}}$, the sequence

$$
0, \overline{\left.\xi^{\left[n_{1}\right.}\right]}, \overline{\xi^{\left[n_{2}\right]}}, \cdots, \overline{\xi^{\left[n_{\ell}\right]}}, \ldots
$$

gives an effective enumeration of the ordinal numbers less than $\alpha_{0}$. Where $n_{i}$ is first $n$ such that $n>n_{i-1}$ and $\overline{\xi^{[n]}} \neq 0$. The notation $\overline{\xi^{[n]}}$ is borrowed from $C$. Kuratowski [2] $]^{11}$.

\section{References}

[1] N. Lusin and P. Novikoff, Choix effectif d'un point dans un complémentaire analytique arbitraire, donné par un crible, Fund, Math., Vol. 25 (1935)

2] C. Kuratowski, Topologie, Vol. 1, 3rd ed., Warszawa, 1952.

1) See, p. 11 and p. 271 . 